\title{
Panorama de las relaciones ínter-coreanas en la era de Kim Jong-un ${ }^{1}$
}

DOI: $10.32870 /$ mycp.v2i3.398

\author{
Ángel Licona Michel ${ }^{2}$ \\ Cintli Ayesa Cárdenas Barajas ${ }^{3}$
}

\section{Resumen}

La muerte de Kim Jong-il en diciembre de 2011 marcó la llegada al poder de su hijo menor Kim Jong-un como el nuevo líder norcoreano, garantizando la sucesión dinástica del régimen iniciado por Kim Il-sung y heredándole la responsabilidad de mantener la continuidad del poder en Corea del Norte, una entidad política polémica por su naturaleza autárquica, dictatorial, discurso beligerante y de desarrollo armamentista.

Así, la llegada de Jong-un guarda implicaciones tanto geopolíticas en lo exterior determinadas por el programa nuclear norcoreano, y cambios en el contexto político de la península, derivados en la postura del nuevo líder hacia el sur y su voluntad de (no) lograr avances en la reconciliación.

En lo que corresponde a las relaciones entre las Coreas, caracterizadas por la búsqueda de reconciliación y reunificación, se han presentado tanto avances como retrocesos oscilantes entre el conflicto y la cooperación, pero permaneciendo siempre en un estado de tensión latente. Por eso, en el análisis multidimensional utilizado para explicar el panorama del estado de las cosas en la península, elementos como la política nacional, las relaciones íntercoreanas diádicas, las políticas de superpotencias y el rol de las organizaciones

1. Artículo recibido el 25 de septiembre de 2012 y dictaminado el 10 de diciembre de 2012.

2. Profesor investigador de la Facultad de Economía y del Centro Universitario de Estudios e Investig-aciones sobre la Cuenca del Pacífico y Centro de Estudios APEC de la Universidad de Colima. ORCID http://orcid.org/0000-0001-6245-8269 Correo electrónico: aliconamichel@gmail.com.

3. Egresada de la Licenciatura en Relaciones Internacionales de la Universidad de Colima. Correo electrónico: cintlicar@hotmail.com. 
internacionales son clave para entender de forma integral el curso presente y futuro de las relaciones entre las Coreas.

En ese sentido, 2012 es un año decisivo para ambas Coreas. Mientras que en Corea del Norte los principales retos para Kim Jong-un son conservar la aprobación en lo interno y mantenerse en lo externo, con el objetivo de reforzar su poder con el respaldo de actores clave; en Corea del Sur se realizarán en diciembre las décimo octavas elecciones presidenciales, lo que representará un cambio tanto de liderazgo como de postura hacia el norte.

Palabras clave: relaciones ínter-coreanas, negociación, superpotencia, inestabilidad política, reunificación, manejo de conflictos, posturas internas.

\section{Abstract}

The demise of Kim Jong-il in December of 2011 meant the emergence of his youngest son Kim Jong-un as North Korea's new leader, guarantying the regime's dynastic succession since Kim Il-sung's decease and inheriting Jongun the responsibility of continuity maintenance of North Korea, a polemic political entity for its self-reliance nature, dictatorship, belligerent discourse and nuclear and missile programs.

Thus, Jong-un's ascent takes over geopolitics implications at external level- determined by North Korea's nuclear program- and changes in the peninsula's political context- determined by the new leader's politics toward the south and his will of achieving paths of reconciliation.

Since the division of the peninsula in 1945, progress and setbacks can be observed in inter-Korean relations- characterized by the permanent seeking of reconciliation and reunification- oscillating from conflict to cooperation, and thus maintaining a status of latent conflict. As a result of that situation, a multidimensional analysis shapes the panorama of inter-Korean reality by introducing key elements for a wider understanding of the present and future of inter-Korean relations: the local level; inter-Korean dyadic level; role of Great Power Politics in Northeast Asia and international organization.

In this regard, the year 2012 is a decisive one for both Koreas. While the main challenges of North Korea's new leadership are the maintenance of internal approval of elites and survival of the regime in light of international pressures, In South Korea the $18^{\text {th }}$ presidential election is coming, which means that a possible polity change towards North Korea will take part of the panorama. 
Keywords: inter-Korean relations, negotiation, superpowers, political instability, reunification, conflict management, internal polity.

\section{Introducción}

Desde la división de la península coreana en 1945 hasta la fecha, el panorama de las relaciones ínter-coreanas - caracterizadas por la búsqueda de reconciliación para lograr la unificación de la nación cultural coreana- ha presentado avances y retrocesos, mismos que han estado altamente determinados por factores internos como las posturas políticas de los dirigentes de ambas Coreas, que oscilan entre el conflicto y la cooperación, y factores externos delimitados por las implicaciones estratégicas sujetas al campo ideológico y de seguridad de potencias regionales como China, Japón y Rusia e internacionales como Estados Unidos, ante cualquier cambio de postura en las Coreas (Chung, 2001).

El hecho de que las dos Coreas sigan divididas (República de Corea [Corea del Sur] y República Popular Democrática de Corea [Corea del Norte]) en la etapa de posguerra fría y que en apariencia la misma lógica de Guerra Fría parezca prevalecer, por la intervención de potencias regionales y mundiales, mientras en paralelo se desarrollen otros elementos como la emergencia de proyectos conjuntos de cooperación económica y relaciones comerciales, constituye una de las paradojas para los estudiosos de Corea: resulta un franco reto encontrar un marco teórico que explique el caso ínter-coreano.

En la búsqueda de un sustento teórico que pudiese explicar de forma integral el caso coreano, se encontró el enfoque de manejo de conflictos (EMC) propuesto por Samuel S. Kim y Abraham Kim, que aplicado a las Coreas se centra en cuatro marcos de análisis que arrojan un escenario general del estado de las cosas en la península (lo interno, lo ínter-coreano, el rol de las potencias y de las organizaciones internacionales). A su vez, los planteamientos de Samuel y Abraham Kim son identificados como parte del paradigma de la Sociedad Mundial en Relaciones Internacionales, que a grandes rasgos señala que las relaciones internacionales no se corresponden con el modelo interestatal sino que con los fenómenos de interdependencia y cooperación surgen nuevos actores que reducen el papel del Estado como entidad soberana $\mathrm{y}$ hacen de la comunidad internacional una sociedad mundial.

Siguiendo un enfoque sistémico, se determina que lo interno y el papel de las potencias son los principales factores que dan forma a los procesos de acercamiento o alejamiento en el monitoreo de las relaciones ínter-coreanas. 
Por lo anterior, en el trabajo se analizan los factores sistémicos y subsistémicos que - producto de los años - caracterizan a las relaciones entre las Coreas. Se contempla la emergencia de nuevos actores que contribuirían al avance o estabilización del proceso de paz. De igual manera se reflexiona sobre la postura oficial del gobierno surcoreano, encabezado por Lee Myungbak, hacia Corea del Norte en el contexto de lo que hasta ahora han revelado ser las relaciones ínter-coreanas con el nuevo líder Kim Jong-un.

El objetivo del trabajo es determinar si el nuevo liderazgo representa continuidad o cambio para lograr acuerdos, proyectos conjuntos y eventualmente dejar de lado los elementos beligerantes que mantienen los niveles de conflicto latentes entre las dos Coreas.

\section{Sustento teórico}

A partir de la premisa planteada por José Luis León (2009) de que el análisis de la realidad ínter-coreana constituye un verdadero laboratorio para las ciencias sociales, en específico para la historia, economía, ciencia política y relaciones internacionales, se afirma que independientemente de la ciencia social desde la que se observen ciertos fenómenos en la península, los preceptos teóricos utilizados se siguen ajustando a la lógica de la Guerra Fría y sus legados realistas, principalmente porque a simple vista permanece la injerencia de potencias en lo externo y los sistemas antagónicos en lo interno.

Independientemente del enfoque teórico utilizado, lo cierto es que las Coreas constituyen para las relaciones internacionales un caso atípico de estudio (Kim, 2004) pues sus relaciones se siguen dando en términos de Estados de facto y no de jure. El hecho de no haber firmado un tratado de paz al finalizar la Guerra de Corea (1953) hace que sus milicias sigan en pie de lucha ante cualquier provocación y aún no haya reconocimiento mutuo oficial (Kim, 2004); ambos Estados constituyen una misma nación cultural, es decir, no son étnicamente diferentes, comparten la misma lengua e historia.

A raíz de la atipicidad del caso, para este trabajo tomamos la teoría de "manejo de conflicto", aplicado al caso ínter-coreano por el politólogo Samuel Kim y el analista de seguridad nacional Abraham Kim (2003); ambos autores se apoyan en estudios de coreanistas como Scott Snyder, Charles Armstrong, Moon Chung-in, Roh Jeong-ho y teóricos como John A. Vázquez, Alexander L. George, entre otros. 
Para Samuel y Abraham Kim, el enfoque de manejo de conflicto (EMC) viene a llenar un vacío de algunas teorías que no tomaban en cuenta las partes prácticas de sus objetos de análisis. De esta manera, el EMC representa una convergencia entre los enfoques teóricos y prácticos de los conflictos, ante la necesidad de mostrar un mapa más integrado, porque, según ambos autores, ya es ampliamente aceptado que las causas de los conflictos no proceden de una sola fuente ni tienen una única solución. Así, se parte de análisis multiniveles y multi-causales (Kim, 2004).

En el contexto internacional de posguerra fría, debido a los fenómenos de globalización al exterior y localización al interior de los países, se observó que la proliferación de conflictos se originó más al interior de los Estados que entre ellos, lo que significa un giro en la conceptualización del término conflicto, visto como una serie de acciones que afectan condiciones de fondo. Por eso, el EMC sirve para explicar que diferentes niveles de las relaciones interestatales e intra-estatales tengan importancia para la diplomacia preventiva (regulación, mitigación y contención de conflictos) como solución a la cultura de reacción (Kim, 2004).

Además, Samuel Kim (2004) afirma que aunque las relaciones actuales entre las Coreas no son muy diferentes a aquellas del fin de la Guerra Fría, su relevancia sigue

Independientemente de la ciencia social desde la que se observen ciertos fenómenos en la península, los preceptos teóricos utilizados se siguen ajustando a la lógica de la Guerra Fría y sus legados realistas, principalmente porque a simple vista permanece la injerencia de potencias en lo externo y los sistemas antagónicos en lo interno creciendo por el involucramiento de actores domésticos, regionales y globales. La afirmación anterior sugiere que las dinámicas de formación de conflicto en la península han sido más endógenas que exógenas, una razón más para estudiar a ambas Coreas en términos multiniveles.

La nación coreana dividida es un caso particular explícito de un conflicto que emerge en distintos niveles y por diferentes lógicas, por lo tanto, se puede notar que cuatro dinámicas establecidas caracterizan el análisis de la península coreana basado en el manejo del conflicto: 
1. Política a nivel local,

2. Relaciones ínter-coreanas diádicas,

3. Las continuadas políticas de las superpotencias,

4. Las organizaciones internacionales.

En relaciones internacionales, el EMC se circunscribe a los enunciados del llamado paradigma de la sociedad mundial o de la interdependencia compleja, que descrito por Celestino del Arenal (2007) caracteriza al sistema internacional como una sociedad mundial, producto del fenómeno de interdependencia y cooperación, como consecuencia del desarrollo social, económico, científicotécnico y comunicacional en el mundo.

De esta manera, según el paradigma de la sociedad mundial, la tradicional separación y distinción entre la esfera interna e internacional ha desaparecido y el fenómeno de la interdependencia, con la necesidad de atender demandas de desarrollo económico y social ha obligado al Estado a abrirse al exterior, restringiendo así su margen de autonomía, haciendo imposible la separación entre política interna e internacional y con ello la explicación de acontecimientos en términos exclusivamente políticos y militares (Del Arenal, 2007: 33-34).

\section{Método}

Los elementos del enfoque de manejo de conflictos se transportaron hacia una metodología donde cada elemento es considerado un componente de un todo interdependiente. En el caso de las relaciones ínter-coreanas se encontró que el rol de las superpotencias y la política local son los elementos que repercuten en mayor medida para la conformación del panorama de la península. Sin embargo, las relaciones diádicas y las organizaciones internacionales no dejan de incidir, de una u otra forma, en el sistema.

\section{Panorama ínter-coreano}

Los acontecimientos históricos que han permitido identificar los marcos de análisis para explicar los fenómenos inherentes a la realidad ínter-coreana también han permitido identificar casos específicos que enriquecen a los mismos marcos de análisis, pero cuyo análisis por separado significa introducirse en contextos que conllevan estudios más amplios y especializados, a los cuales no se llega en este trabajo. 


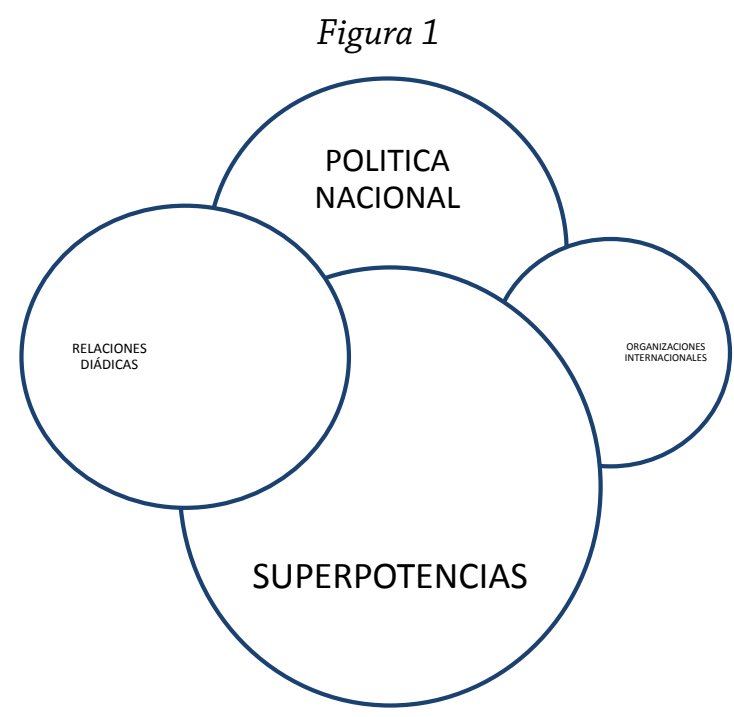

Elaboración propia a partir del modelo de manejo de conflictos aplicado a la península coreana, de Samuel S. Kim (2004) y Abraham Kim (2003).

En la tabla 1, se mencionan a grandes rasgos los factores que conforman a cada elemento sistémico, que en cierta forma impactan el panorama de las relaciones en la península.

\section{Tabla 1}

Los elementos del panorama inter-coreano

\begin{tabular}{|c|c|c|c|}
\hline $\begin{array}{l}\text { Elemento } \\
\text { sistémico }\end{array}$ & \multicolumn{3}{|c|}{ Descripción histórico-contextual } \\
\hline \multirow{3}{*}{ Nivel local } & \multirow[b]{2}{*}{ Político } & \multicolumn{2}{|c|}{$\begin{array}{l}\text { - Sistema político y económico de ambas Coreas son an- } \\
\text { tagónicos (capitalismo-socialismo). }\end{array}$} \\
\hline & & \multicolumn{2}{|c|}{$\begin{array}{l}\text { Ambos sistemas han intentado clamar legitimidad y } \\
\text { han tenido que negar al otro Estado como similar: com- } \\
\text { petencia por superioridad económica, militar, política y } \\
\text { reconocimiento internacional. }\end{array}$} \\
\hline & $\begin{array}{l}\text { Elementos de } \\
\text { cambio socio- } \\
\text { político }\end{array}$ & $\begin{array}{l}\text { En Corea del } \\
\text { Sur }\end{array}$ & $\begin{array}{l}\text { - Más libertad de acceso a información } \\
\text { sobre Corea del Norte desde la Nordpo- } \\
\text { litik de Roh Tae-woo; impacto positivo } \\
\text { de la Sunshine Policy de Kim Dae-jung. }\end{array}$ \\
\hline
\end{tabular}


Ángel Licona Michel y Cintli Ayesa Cárdenas Barajas

\begin{tabular}{|c|c|c|c|}
\hline Elemento & \multicolumn{3}{|c|}{ Descripción histórico-contextual } \\
\hline \multirow[t]{2}{*}{ Nivel local } & \multirow[t]{2}{*}{$\begin{array}{l}\text { Elementos de } \\
\text { cambio socio- } \\
\text { político }\end{array}$} & \multirow[t]{2}{*}{$\begin{array}{l}\text { En Corea del } \\
\text { Sur }\end{array}$} & $\begin{array}{l}\text { - Brecha generacional en población sur- } \\
\text { coreana: las generaciones más viejas } \\
\text { son escépticas sobre posibilidades de } \\
\text { compromiso y acuerdos, mientras que } \\
\text { las más jóvenes esperan menos bene- } \\
\text { ficios de los acuerdos y posible reuni- } \\
\text { ficación. Pero en ambas se tiene cierta } \\
\text { empatía hacia el vecino del norte y su } \\
\text { situación socio-económica. }\end{array}$ \\
\hline & & & $\begin{array}{l}\text { - Sigue vigente la Ley de Seguridad Na- } \\
\text { cional. }\end{array}$ \\
\hline \multirow{4}{*}{ Nivel local } & \multirow{4}{*}{$\begin{array}{l}\text { Elementos de } \\
\text { cambio socio- } \\
\text { político }\end{array}$} & $\begin{array}{l}\text { En Corea del } \\
\text { Sur }\end{array}$ & $\begin{array}{l}\text { - Papel de la iniciativa privada: Hyundai } \\
\text { Asan como pionera de proyectos con- } \\
\text { juntos ínter-coreanos como el Comple- } \\
\text { jo Industrial de Kaesong y el ya can- } \\
\text { celado proyecto turístico del Monte } \\
\text { Kumgang. }\end{array}$ \\
\hline & & \multirow[t]{3}{*}{$\begin{array}{l}\text { En Corea del } \\
\text { Norte }\end{array}$} & $\begin{array}{l}\text { Military First Politics e idea Juche, dos } \\
\text { de las bases teóricas y marcos de ac- } \\
\text { ción en las que se sostiene el régimen } \\
\text { norcoreano. Ambas restringen libertad } \\
\text { de información sobre Corea del Sur. }\end{array}$ \\
\hline & & & $\begin{array}{l}\text { - Programa nuclear y de misiles de largo } \\
\text { alcance: resultado de Military First Po- } \\
\text { litics. }\end{array}$ \\
\hline & & & $\begin{array}{l}\text { - El crecimiento del mercado negro, } \\
\text { contrabando de tecnología, tipos de } \\
\text { cambio, personas y diplomacia cultural } \\
\text { surcoreana en el Hallyu han llevado a } \\
\text { un cambio de actitud en la población. }\end{array}$ \\
\hline \multirow[t]{2}{*}{ Nivel diádico } & \multirow{2}{*}{$\begin{array}{l}\text { Acuerdos } \\
\text { ínter- } \\
\text { coreanos más } \\
\text { importantes }\end{array}$} & \multicolumn{2}{|c|}{$\begin{array}{l}\text { Comunicado conjunto del } 4 \text { de julio de 1972: las Coreas con- } \\
\text { sagran tres principios imprescindibles para la reunifica- } \\
\text { ción: 1) alcanzar la meta sin interferencias externas; 2) } \\
\text { lograr unidad a través de medios pacíficos; 3) culminar el } \\
\text { proceso rebasando las diferencias en los proyectos, ideo- } \\
\text { logías y sistemas. }\end{array}$} \\
\hline & & \multicolumn{2}{|c|}{$\begin{array}{l}\text { Acuerdo básico de } 1991 \text { "Acuerdo de Reconciliación, No Agre- } \\
\text { sión, Intercambio y Cooperación". En su artículo } 5 \text { estipula } \\
\text { que deberán acatar el acuerdo de Armisticio Militar actual } \\
\text { hasta que se llegue a la paz. }\end{array}$} \\
\hline
\end{tabular}


Panorama de las relaciones inter-coreanas en la era de Kim Jong-un

\begin{tabular}{|c|c|c|}
\hline $\begin{array}{l}\text { Elemento } \\
\text { sistémico }\end{array}$ & \multicolumn{2}{|c|}{ Descripción histórico-contextual } \\
\hline \multirow{8}{*}{ Nivel diádico } & $\begin{array}{l}\text { Acuerdos } \\
\text { ínter- } \\
\text { coreanos más } \\
\text { importantes }\end{array}$ & $\begin{array}{l}\text { Declaración Conjunta de 2000. Esfuerzos autónomos de las } \\
\text { dos Coreas por la reunificación basada en el común reco- } \\
\text { nocimiento de similitudes, rápida resolución de asuntos } \\
\text { referentes a las familias separadas y los prisioneros poli- } \\
\text { ticos, balanceado desarrollo económico, diálogos y consul- } \\
\text { tas entre autoridades gubernamentales. }\end{array}$ \\
\hline & \multirow[b]{2}{*}{$\begin{array}{l}\text { Relaciones } \\
\text { comerciales }\end{array}$} & $\begin{array}{l}\text { - Se dan desde fines de la década de los } 80 \text { (Kim y Win- } \\
\text { ters, 2004) en base al criterio de "Processing On Co- } \\
\text { mission Trade" (POC) en que compañías surcoreanas } \\
\text { importan materia prima de Corea del Norte y después } \\
\text { exportan productos acabados o semi-acabados. }\end{array}$ \\
\hline & & $\begin{array}{l}\text { - Las relaciones comerciales las lidera Corea del Sur, y Co- } \\
\text { rea del Norte las acepta para crear vínculos funcionales } \\
\text { en manejo de conflictos, paz y reunificación. Por estos } \\
\text { vínculos funcionales Corea del Sur ha pasado por alto } \\
\text { las amenazas de Corea del Norte para continuar promo- } \\
\text { viendo comercio e inversión (Kim, 2004). }\end{array}$ \\
\hline & \multirow{4}{*}{$\begin{array}{l}\text { Proyectos } \\
\text { conjuntos }\end{array}$} & $\begin{array}{l}\text { - Conexión de caminos y vías del tren entre ambas Co- } \\
\text { reas (proyecto iniciado en } 2002 \text { y completado en 2004, } \\
\text { pero no hay tráfico ínter-coreanos significativo). }\end{array}$ \\
\hline & & $\begin{array}{l}\text { - Proyecto turísitco del Monte Kumgang (abierto en } \\
1998 \text { y cerrado oficialmente en 2011). }\end{array}$ \\
\hline & & $\begin{array}{l}\text { - Complejo Industrial de Kaesong (iniciado a fines de } \\
2004 \text {, sigue sus funciones hasta hoy). }\end{array}$ \\
\hline & & $\begin{array}{l}\text { Ayuda humanitaria por parte de Corea del Sur hacia el } \\
\text { norte, por los constantes desabastecimientos de gra- } \\
\text { nos, cereales, leguminosas, entre otros. }\end{array}$ \\
\hline & $\begin{array}{l}\text { La carencia de } \\
\text { efectos legales }\end{array}$ & $\begin{array}{l}\text { Para el jurista Roh Jeong-ho (Kim,2004) los acuerdos ín- } \\
\text { ter-coreanos y relaciones bilaterales descansan en una ilu- } \\
\text { soria fundación legal debido a que nunca se ha concretado } \\
\text { un tratado de paz permanente. El Acuerdo del Armisticio } \\
\text { de } 1953 \text { permanece como el único acuerdo legal que rige } \\
\text { a las relaciones diádicas lo que hace a las relaciones bila- } \\
\text { terales más complejas y ambiguas de lo que pudiesen ser. }\end{array}$ \\
\hline \multirow{4}{*}{$\begin{array}{l}\text { Superpotencias } \\
\text { en el este de } \\
\text { Asia }\end{array}$} & \multirow{2}{*}{ EEUU } & - Intenta desmantelar el desarrollo nuclear norcoreano. \\
\hline & & - Presencia militar en Corea del Sur. \\
\hline & \multirow[b]{2}{*}{ China } & - Apoyo al régimen norcoreano. \\
\hline & & $\begin{array}{l}\text { - Intento de incentivar apertura de mercados al estilo } \\
\text { chino en corea del norte. }\end{array}$ \\
\hline
\end{tabular}


Ángel Licona Michel y Cintli Ayesa Cárdenas Barajas

\begin{tabular}{|c|c|c|}
\hline $\begin{array}{l}\text { Elemento } \\
\text { sistémico }\end{array}$ & \multicolumn{2}{|c|}{ Descripción histórico-contextual } \\
\hline \multirow{3}{*}{$\begin{array}{l}\text { Superpotencias } \\
\text { en el este de } \\
\text { Asia }\end{array}$} & \multirow[b]{2}{*}{ Rusia } & - Proyecto de conexiones de tren transiberiano. \\
\hline & & $\begin{array}{l}\text { - Proyectos de abastecimiento de fuentes energéticas } \\
\text { como gas natural. }\end{array}$ \\
\hline & Japón & - Más enfocado a observar realidad ínter-coreana. \\
\hline $\begin{array}{l}\text { Organizaciones } \\
\text { internacionales }\end{array}$ & \multicolumn{2}{|c|}{$\begin{array}{l}\text { Este elemento juega un rol indirecto y limitativo en el proceso de paz. } \\
\text { Sin embargo, algunas organizaciones han ofrecido foros de convergen- } \\
\text { cia entre ambos estados, en el que se interactúa para la creación de vín- } \\
\text { culos funcionales, lo que puede reforzar los avances hechos y presentar } \\
\text { oportunidades para nuevas iniciativas. }\end{array}$} \\
\hline
\end{tabular}

Fuente: elaboración propia con base en información consultada en la parte introductoria del libro Inter-Korean relations: problems and prospects de Samuel S. Kim (2004).

\section{Postura actual de Corea del Sur hacia Corea del Norte}

Como se evidenció en la tabla 1 , en el transcurso de la historia las posturas de las Coreas, tanto en nivel interno como diádico han cambiado gradualmente. A mediados del siglo xx la lucha de ambas era por la legitimidad —en esos momentos las diferencias socio-económicas de las dos Coreas no eran tan marcadas como ahora-y no se llegaba a ningún acuerdo importante porque ambos sistemas se desconocían mutuamente y clamaban ser las mejores opciones para la península reunificada; por lo tanto, aunque deseaban la reunificación como fin último, los medios eran violentos, pues la intolerancia recíproca inherente a los dos sistemas antagónicos imperantes dictaba el dominio de un sistema sobre el otro. La guerra o la espera del colapso del algún lado de la península eran los imperativos que dominaban el escenario ínter-coreano.

A fines del siglo xx la relativa paridad económica de ambas Coreas ya había desaparecido, porque los cambios en el mismo sistema internacional -en específico el fin de la Guerra Fría y con ello el colapso de la Unión Soviéticahabían dejado desprotegida a Corea del Norte; mientras que por otro lado, Corea del Sur - como Estado "desarrollista" capitalista - se constituía como una de las economías más fuertes de Asia (Chung, 2001).

El inicio del siglo XXI representó un parteaguas para las relaciones íntercoreanas, por el ambiente de apertura en ambos países, caracterizado por el respeto mutuo hacia los diferentes sistemas políticos, la comprensión de las distintas situaciones económicas y una voluntad real de lograr la reunificación gradual, no forzada por la imposición de un sistema político-ideológico como se manejó en el siglo previo. 
En el caso específico de Corea del Sur, el cambio de postura hacia el norte tuvo nombres y apellidos, se le llamó "Sunshine Policy", una política de compromiso consistente en brindar apoyo económico incondicional e incentivos hacia Corea del Norte con el fin de que la situación de aquel país -en decadencia desde fines de la década de 1970 por la caída del socialismo real y reconfiguración del sistema internacional a favor del capitalismo- mejorara hasta igualar gradualmente a Corea del Sur. Esta postura de compromiso se mantuvo en el sur desde 2000 hasta 2007, bajo los periodos de gobierno de Kim Dae-jung y Roh Moo-hyun (Kim, 2004).

Corea del Norte, por su parte, bajo la dirigencia de Kim Jong-il (ascendido al poder en 1994) demostró ser pragmática y desarrollar un método de supervivencia donde aprovechaba la ayuda internacional, enfocada a estimular las reformas económicas para consolidar su liderazgo con base en una política de militarización y desarrollo de tecnología nuclear (Military First Politics), esto último provocó un dilema en Corea del Sur acerca de si debería o no continuar cooperando incondicionalmente con su vecino, así como también una consternación mundial por considerar a este país un peligro para la estabilidad regional.

Por eso, en la sucesión presidencial surcoreana de 2008, el presidente Lee Myung-bak (2008-2013, véase anexo 1) condicionó la ayuda a Corea del Norte en la medida de que hiciera avances tangibles para desnuclearizarse. Ante esta postura Kim Jong-il respondió suspendiendo el diálogo oficial entre ambas Coreas, lanzando agresiones verbales, entre otras acciones, incluso una turista surcoreana en el complejo turístico del Monte Kumgang fue muerta por un soldado norcoreano (véase anexo 2). Las negociaciones de reconciliación se estancaron.

Lee Myung-bak enfatizó en el discurso inaugural de su gobierno que su actitud hacia las relaciones ínter-coreanas sería pragmática, no ideológica, representada en la "visión 3000", donde a cambio de la desnuclearización norcoreana, con su regreso a las negociaciones a seis bandas, ${ }^{4}$ instrumentaría

4. Estas negociaciones surgieron como instancia de diálogo luego de que Corea del Norte se retirara del Tratado de No Proliferación Nuclear de la ONU en 2003. En los diálogos participaban seis países: Corea del Sur, Corea del Norte, China, Japón, Rusia y Estados Unidos con el objetivo de que el gobierno de Pyongyang cerrara sus instalaciones nucleares a cambio de ayuda internacional. De 2003 a 2007 se han llevado a cabo seis rondas de conversaciones, pero tras algunos impasses diplomáticos entre los países participantes y el Consejo de Seguridad de la ONU, Corea del Norte decidió abandonar unilateralmente las rondas de negociaciones y reabrir sus instalaciones nucleares (BCN, 2012: 20). 
un plan para proveer asistencia a los norcoreanos, con el fin de elevar el ingreso per cápita a 3,000 dólares (en 2011 según CIA World Factbook el PIB per cápita norcoreano era de 1,800 dólares) en 10 años (Manyin y Nanto, 2010).

Otra estrategia de Myung-bak fue "La Nueva Iniciativa de Paz para la Península Coreana" (Ministerio de Unificación, 2010) también llamada "el gran regateo". Esta iniciativa reza que en la medida que Corea del Norte desista de sus ambiciones nucleares, el sur asistirá a los norcoreanos en cinco áreas principales: economía, educación, finanzas, infraestructura industrial y calidad de vida.

En detalle, la postura de Lee Myung-bak hacia el norte, según el Ministerio de Unificación (2010) se basa en tres aspectos: crear una nueva estructura de paz en la península a través de la desnuclearización de Corea del Norte, construir una comunidad económica común (reforzar los proyectos de cooperación económica existentes) y resolver asuntos humanitarios.

Asimismo, el Ministerio de Unificación deja claro que a pesar de las provocaciones norcoreanas (véase anexo 2) el gobierno surcoreano permanecerá inamovible, continuando la promoción de los proyectos económicos y la asistencia humanitaria. La administración de Lee Myung-bak afirma que el diálogo tiene que ser sostenido, no suspendido de forma unilateral, en continuación con el círculo vicioso ínter-coreano de seguir evadiendo asuntos nucleares, estratégicos y políticos, pero alentando la cooperación económica, relaciones comerciales y asistencia humanitaria sin concretar nada estable.

En la otra cara de la moneda, en 2008 el estado de salud del Kim Jong-il se vio deteriorado y despertó suspicacias internacionales sobre el inicio de un posible proceso de transición. En 2009 se comenzó a vislumbrar que el sucesor de Kim Jong-il sería Kim Jong-un, hijo menor del líder norcoreano.

Se dice que Jong-un antes de detentar el poder era un personaje poco conocido incluso entre los norcoreanos. Según las agencias internacionales de noticias, se cree tiene alrededor de 30 años, estudió en Suiza, habla varios idiomas, tiene el carácter de su padre e incluso "comparte algunos de sus problemas de salud. Ha sido reportado que tiene diabetes y enfermedades del corazón" (BBC, 2011).

El proceso de sucesión se aceleró en 2010, con dos importantes agresiones norcoreanas, una dudosa y otra no (véase anexo) que según analistas marcaron el preludio de lo que vendría después con el incremento de apariciones públicas del joven Kim y nombramientos privilegiados como general de cuatro estrellas y vicepresidente de la Comisión Militar Central (Korea Economic Institute, 2012). 
El 17 de diciembre de 2011 ocurrió lo predecible, se anunció la muerte de Kim Jong-il y días más tarde, el 26 de diciembre, el periódico norcoreano Rodong Sinbum anunció el nombramiento de Kim Jong-un como presidente de la Comisión Militar Central y líder supremo del país. Con lo anterior se dio inicio a la era Kim Jong-un (Manyin, 2011).

\section{Panorama ínter-coreano bajo el gobierno de Kim Jong-un}

En lo que va del recién establecido régimen norcoreano, se pueden observar dos tendencias relevantes: al interior, los líderes políticos detrás de Kim Jong-un están preocupados y ocupados en mostrar la unidad de la nación y la lealtad de las élites hacia el gobernante neófito (Hamisevicz, Yun, O'Carroll y Stangarone, 2012) mientras que al exterior, lo sobresaliente recae en la expectativa de que el joven Kim tenga disposición de refundar el diálogo a seis bandas por un lado, y por el otro acceder a retomar las conversaciones bilaterales con Seúl.

Estas realidades permiten entender la estructura actual de las relaciones ínter-coreanas. Sin embargo, además del rol de las potencias y del plano sociopolítico interno de las Coreas, hay elementos esenciales delineados en el enfoque de manejo de conflictos, ya antes señalados en este trabajo, que no pueden ser dejados de lado si se quiere obtener un cuadro más completo del asunto. Por lo cual, para una mejor comprensión del entramado de factores circunscritos al enfoque aplicado, se elaboró tabla 2.

Tabla 2

Panorama ínter-coreano bajo Kim Jong-un

\begin{tabular}{|c|c|c|c|}
\hline Elementos de análisis & & Corea del Sur & Corea del Norte \\
\hline \multirow[t]{2}{*}{ Situación interna } & \multirow{2}{*}{$\begin{array}{l}\text { Plano } \\
\text { gubernamental }\end{array}$} & Lee Myung-bak & Kim Jong-un \\
\hline & & $\begin{array}{l}\text { Diplomacia } \\
\text { condicionada } \\
\text { hacia Pyongyang. }\end{array}$ & $\begin{array}{l}\text { Estancamiento } \\
\text { diplomático en } \\
\text { negociaciones con el } \\
\text { Sur por permanencia de } \\
\text { postura rígida y cerrada } \\
\text { al diálogo. }\end{array}$ \\
\hline
\end{tabular}


Ángel Licona Michel y Cintli Ayesa Cárdenas Barajas

\begin{tabular}{|c|c|c|c|}
\hline \multicolumn{2}{|l|}{ Elementos de análisis } & \multirow[b]{2}{*}{$\begin{array}{l}\text { Corea del Sur } \\
\text { - Inquietud ante } \\
\text { inexperiencia } \\
\text { política de Kim } \\
\text { Jong-un. }\end{array}$} & \multirow{2}{*}{$\begin{array}{l}\text { Corea del Norte } \\
\text { - Postura de no } \\
\text { negociación con Lee } \\
\text { Myung-bak. }\end{array}$} \\
\hline Situación interna & $\begin{array}{l}\text { Plano } \\
\text { gubernamental }\end{array}$ & & \\
\hline & & $\begin{array}{l}\text { Dependencia de } \\
\text { las decisiones de } \\
\text { Estados Unidos. } \\
\end{array}$ & $\begin{array}{l}\text { - Preservación de políticas } \\
\text { de Kim Jong-il: "Songun", } \\
\text { "Juche". }\end{array}$ \\
\hline & & $\begin{array}{l}\text { Reiterada invi- } \\
\text { tación al Norte } \\
\text { para reanudación }\end{array}$ & $\begin{array}{l}\text { Aceptación del nuevo } \\
\text { líder por parte de las } \\
\text { elites. }\end{array}$ \\
\hline & & de diálogo. & $\begin{array}{l}\text { Dependencia hacia elites } \\
\text { norcoreanas, en espe- } \\
\text { cífico a Jang Song-taek } \\
\text { (Director Administrativo } \\
\text { del Partido de los Traba- } \\
\text { jadores Coreanos) y Kim } \\
\text { Kyong-hui (Directora de } \\
\text { la División de Industria } \\
\text { Ligera del Partido de los } \\
\text { Trabajadores). }\end{array}$ \\
\hline & Plano social & $\begin{array}{l}\text { La tensión ínter- } \\
\text { coreana redujo } \\
\text { el optimismo } \\
\text { de la opinión } \\
\text { pública sobre } \\
\text { perspectivas de } \\
\text { reunificación. }\end{array}$ & $\begin{array}{l}\text { Desconcierto ante } \\
\text { falsedad del discurso que } \\
\text { aseguraba la condición } \\
\text { de opresión y pobreza } \\
\text { en el Sur (por acceso a } \\
\text { la diplomacia cultural } \\
\text { surcoreana). }\end{array}$ \\
\hline & & & $\begin{array}{l}\text { Padecimiento de } \\
\text { hambre, pobreza, } \\
\text { violación a los derechos } \\
\text { humanos. }\end{array}$ \\
\hline Relaciones diádicas & Acuerdos lograd & & \\
\hline $\begin{array}{l}\text { Rol de las } \\
\text { superpotencias. }\end{array}$ & & $\begin{array}{l}\text { Estados Unidos: } \\
\text { presencia de } \\
\text { apoyo militar en } \\
\text { la frontera con el } \\
\text { norte. } \\
\text { Representa para } \\
\text { el Sur una guía a } \\
\text { seguir en cuanto a } \\
\text { las decisiones con } \\
\text { Pyongyang }\end{array}$ & $\begin{array}{l}\text { Estados Unidos: } \\
\text { Nueva apertura para } \\
\text { la reanudación de las } \\
\text { negociaciones a seis } \\
\text { bandas y la gradual } \\
\text { desnuclearización. }\end{array}$ \\
\hline
\end{tabular}


Panorama de las relaciones inter-coreanas en la era de Kim Jong-un

\begin{tabular}{lll}
\hline Elementos de análisis & Corea del Sur & Corea del Norte \\
\hline & China: Mediador. Teme inestabilidad en su \\
frontera por las consecuencias que le traería el \\
colapso. Teme la unificación porque reduciría \\
su dominio en el Norte, abriendo el camino a \\
postulados pro capitalistas.
\end{tabular}

Fuente: elaboración propia con base en artículos de Pablo Kummetz, Mark E. Mayin, Nicholas Hamisevicz et al., así como del Ministerio de Unificación de Corea, CNN, El Universal, El Mundo y RPP Noticias de Perú.

Si se representara gráficamente la importancia de los cuatro elementos presentes en la tabla 2 por su nivel de incidencia en el escenario de las Coreas bajo el régimen de Kim Jong-un, la figura quedaría de la siguiente manera:

Como se puede observar, el plano interno juega el rol más trascendente. Para el caso norcoreano, es indispensable el equilibro político interior, lo cual, si bien resulta complicado para todo Estado, lo es más para uno que tiene que compartir el poder entre todos y cada uno de los miembros que conforman la elite (véase anexo 1 y 3); sin embargo, los especialistas aseguran que el trabajo más complicado ya fue realizado previo al fallecimiento de Kim Jong-il, quien efectuó una labor de acondicionamiento dentro del país (entre otras cosas, realizó un manejo efectivo de las facciones y revigorizó el partido) para 
Figura 2

Régimen Kim Jong-un con el planteamiento EMC

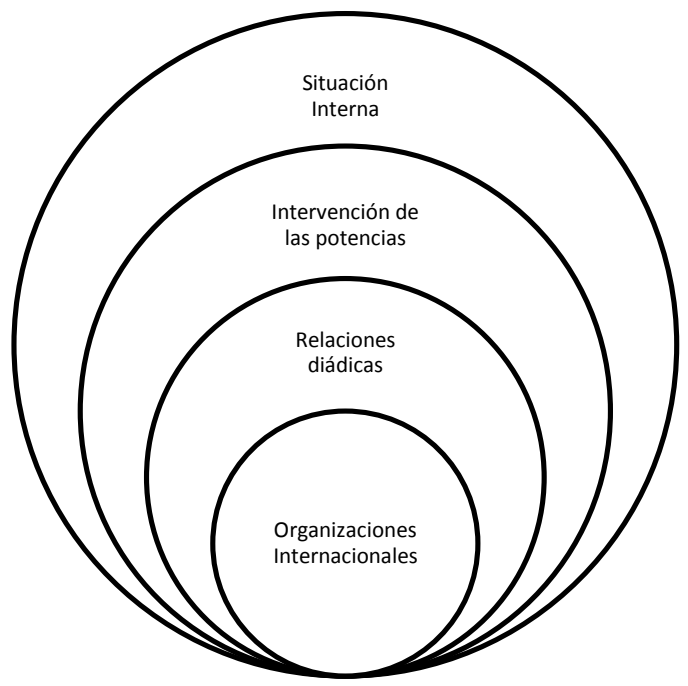

asegurar una transición más sencilla a su sucesor, lo que simultáneamente permitirá un mayor margen de maniobra en caso de que algunos miembros de la elite no concordaran con la dirección que está tomando la nación (Hamisevicz et al., 2012).

Mientras tanto, en el caso surcoreano, se pronostica que la insistencia para restablecer el diálogo con su vecino del Norte no se irá a la par de la administración de Lee Myung-bak, sino que, con base en los adelantos discursivos de los principales pre candidatos políticos, es posible conjeturar que habrá continuidad del tema en la práctica diplomática.

En lo que concierne al segundo tema de mayor significancia - la relación con las superpotencias-, el gobierno de Kim Jung-un se muestra abierto. Tal actitud, cabe señalar, fue iniciada por el querido líder en 2011 y todo apunta a que tal camino será seguido por su hijo, el gran sucesor, eso se debe, arguye Rodger Baker (2012) a que la entrada de recursos financieros que le proveen al país resulta primordial para mantener a las elites en un estado de confort, ya que tales fondos le permiten "tener un pastel más grande para compartir con los diferentes elementos de la elite” (Baker, 2012). 
Ejemplo de ello es que recibe de China, principalmente, recursos dinerarios enviados directamente al fondo de ingresos de la nación y posteriormente repartidos entre el gobierno y el ejército. Otra evidencia es la reunión en Beijing el pasado 23 de febrero, de la delegación estadounidense y de la representación de Corea del Norte para reactivar las conversaciones a seis bandas. En este encuentro Corea del Norte decidió aceptar una moratoria de desarrollo nuclear y de lanzamiento de misiles a cambio del envío de ayuda alimentaria (Kummetz, 2012).

Para finalizar, la contribución de las relaciones diádicas y el papel de las organizaciones internacionales al nexo ínter-coreano es prácticamente nula, puesto que - tal como se aprecia en la tabla 2- no hay acuerdo alguno, lo cual era de esperarse después de la firme declaración en que Corea del Norte anunció que Corea del Sur y otras naciones no deberían esperar ningún cambio de la nueva administración nacional (El Mundo, 2011). Por otro lado, la ONU y Amnistía Internacional han sido las OI con mayor involucramiento, dirigido a condenar las vejaciones a los derechos humanos cometidas en Corea del Norte. En el corto periodo de tiempo que lleva al mando Kim Jong-un ya se han realizado ejecuciones de desertores y enviado a campos de internamiento a personas vistas como una amenaza para el nuevo régimen (RPP Internacional, 2011).

\section{Reflexiones finales}

El panorama ínter-coreano hoy es más incierto de lo que era en la primera transición de liderazgo en 1994, cuando Kim Jong-il llegó al poder y tanto en la academia como en el plano gubernamental se prevenía el inminente colapso del régimen norcoreano dentro de un plazo de no más de tres años (Kim, 2004). Como esto no sucedió y contrario a las predicciones el régimen continuó e incluso encontró la manera de sobrevivir, ahora que se llevó a cabo la segunda transición con Kim Jong-un, el estudio de ambas Coreas se ha revitalizado y con ello las posturas menos arrebatadas arrojan que por el momento, y gracias a varios factores, Jong-un permanecerá.

De acuerdo con el EMC seguido a lo largo de este trabajo, se concluye que:

1. Las relaciones entre ambas Coreas están altamente influenciadas por la injerencia de las potencias. En el caso norcoreano, su diplomacia varias veces se dirige primero a Estados Unidos (no a Corea del Sur) para negociar el tema nuclear y humanitario. Jong-un no es la excepción. Este escenario 
no rompe con el círculo vicioso conflicto-cooperación-conflicto en que prevalecen a nivel diádico acuerdos económicos y comerciales sin avanzar en aspectos políticos y de seguridad (i.e. el concretar la reconciliación y dejar, de una vez por todas, el estado de confrontación latente).

2. Aunque están presentes las organizaciones internacionales, en realidad no juegan un rol central en las relaciones ínter-coreanas, puesto que el escenario lo domina la lógica de superpotencias, lo que no da cabida a las posturas conciliatorias ofrecidas por las organizaciones.

3. La convergencia de intereses - en mayor o menor medida- de Estados Unidos, China, Japón y Rusia, mantienen el tan sonado "equilibrio de poder" (dichas potencias aún operan bajo la lógica de alianzas ideológicas y de seguridad de la Guerra Fría, oficialmente terminada).

4. Sin embargo, se pueden encontrar atisbos de cambio social en ambas entidades políticas que retan a las tradicionales visiones realistas. En el caso del nivel doméstico, en Corea del Sur resaltan las muestras de simpatía hacia la sociedad norcoreana y menos empatía hacia la injerencia estadounidense, que a decir de muchos, no hace más que seguir prolongando el statu quo de la Guerra Fría. Mientras que en Corea del Norte, gracias a la proliferación de productos de diplomacia cultural surcoreana (Hallyu) en el mercado negro, la percepción hacia Corea del Sur también cambia, más que nada porque se evidencia que la situación en el sur no es como el régimen norcoreano dice que es.

5. Finalmente, el nivel diádico permanece como el elemento más tradicional e inamovible de todos por falta de un marco jurídico que se adapte a las nuevas realidades.

El panorama de las relaciones ínter-coreanas en lo que va de la "era Kim Jongun" puede ser calificado como de continuidad, sin cambios ni replanteamientos lo suficientemente retadores al sistema consolidado bajo Kim Il-sung y Kim Jong-il. En gran parte, este panorama de continuidad se debe a que en el nivel interno, Lee Myung-bak todavía detenta el poder en el sur. La expectativa es que la actitud norcoreana cambie a la par de la administración de Lee. De esta manera, en diciembre de 2012 con un nuevo dirigente electo en el sur, el nexo podría reanudarse y el diálogo bilateral retomarse. Todo depende de quién resulte electo en los próximos comicios. 


\section{Al cierre}

Dada la rapidez con la que han transcurrido acontecimientos económicos, políticos y militares en la península coreana a lo largo de este 2012 y estando a escasas semanas del primer aniversario del ascenso de Kim Jong-un al poder y a tres días de las elecciones del 19 de diciembre en Corea del Sur, decidimos actualizar el artículo sin alterar su estructura.

Esta actualización toma de referencia principal el estudio académico más actual de la era Kim Jong-un, escrito por el catedrático de la Universidad de la Columbia Británica Lynn Hyun-gu (2012), quien con base en estudios tanto cuantitativos $^{5}$ como cualitativos ${ }^{6}$ llega a la conclusión de que el régimen de Jong-un representa más continuidad que cambio tanto a nivel interno como internacional y en relaciones ínter-coreanas. Asímismo, predice que si la administración del nuevo líder continúa al mismo ritmo de este año, fácilmente llegará a su quinto aniversario sin mucho problema.

Según el académico, la revitalización del liderazgo norcoreano, las frecuentes apariciones públicas de Kim Jong-un incluso acompañado de su esposa Ri Sol-ju, sus participaciones en eventos juveniles masivos y apertura de parques de diversiones no representan más que una de las muchas estrategias empleadas por sus antecesores para despertar la simpatía de la población puesto que los controles sociales siguen inflexibles y la libertad de expresión es limitada.

Por otra parte, y en línea con lo que se planteó en el inciso VI de este estudio, las purgas de prominentes oficiales norcoreanos que se han llevado a cabo en últimos meses, no van más allá de las tácticas usadas tanto por Kim Il-sung y Kim Jong-il para consolidar el poder. ${ }^{7}$ En este punto, destaca la remoción total de Ri Yong-ho (véase anexo) en el mes de julio, que causó amplia controversia internacional y levantó muchas dudas acerca de la estabilidad del norte.

5. Indicadores internacionales como el índice de inestabilidad política, índice de riesgo político e indicadores de gobernanza global.

6. Monitoreo del número de apariciones de individuos en eventos con el nuevo líder, seguimiento del orden de aparición en eventos oficiales, apariciones en la agencia coreana de noticias KCNA y recaudación de información de desertores norcoreanos en otros países.

7. Lynn Han Gu documenta la remoción por parte de Kim Jong-un de varios asesores cercanos a su padre, realización de cambio de personal a gran escala y ascenso de puestos a los oficiales más leales. En general, según el académico, los últimos meses 30\% de oficiales del ejército mayores de 70 años han sido reemplazados por oficiales de entre 40 y 50 años. 
Respecto al nivel ínter-coreano y externo, destaca la reapertura del complejo turístico conjunto del Monte Kumgang en noviembre; el aumento considerable de intercambios culturales y deportivos con países del sureste de Asia, así como el rechazo a entrar nuevamente a las negociaciones a seis bandas; las advertencias norcoreanas hechas en los meses de octubre y noviembre a los Estados Unidos sobre la capacidad de su tecnología de misiles de alcanzar suelo estadounidense; el anuncio en noviembre del estado de semi-guerra con Corea del Sur a razón de los ejercicios militares anuales del Sur; la rendición de honores por parte de Kim Jong-un a los soldados norcoreanos que participaron en el bombardeo de la isla de Yeonpyong en noviembre de 2011; el polémico lanzamiento del satélite Unha-3 el 12 de diciembre, entre otras acciones, confirman lo que la comunidad internacional (con sus excepciones) no deseaba de Kim Jong-un: la continuación de intercambios culturales y maniobras diplomáticas en paralelo con amenazas militares, una estrategia de negociación característica de Pyongyang desde 1960.

Kim Jong-un, entonces, no es el reformista esperado y contrario a lo que se pronostica de su administración, el nuevo líder, con sus reformas cambiantes, pero no amenazantes al statu quo norcoreano, sigue reforzando su poder en las mayores entidades administrativas del régimen: el Politburó, la Comisión Militar Central y la Comisión de Defensa Nacional. La clave, según Lynn Hyun-gun, es mantener el equilibrio de poder entre las mayores entidades administrativas para así seguir reforzando la estabilidad interna.

Por tanto, se afirma que son pocas o nulas las posibilidades de un golpe de Estado, intervención de alguna o algunas potencias, negociación de un cambio de régimen político o un levantamiento de masas.

En cambio, Corea del Sur está próxima a elegir nuevo presidente. Hasta el momento, la contienda se definirá entre la candidata de línea conservadora Park Geun-hye, hija del fallecido dictador surcoreano Park Chung-hee y el ex defensor de los derechos humanos, de línea liberal Moon Jae-in, quien también fuera ayudante del fallecido presidente Roh Moo-hyun (Mc Curry, 2012).

La postura de ambos candidatos hacia Corea del Norte y la definición del carácter de las relaciones ínter-coreanas es conciliadora, pero con diferentes matices. Mientras que Park Geun-hye pretende definir su política hacia el norte en base a una nueva "trustpolitik" entre ambas Coreas - no sin antes demandar a Pyongyang el abandono de su programa nuclear y de misiles (de acuerdo a la política actual de Lee Myung-bak)—, Moon Jae-in ofrecerá a Kim Jong-un "dialogo incondicional” (Mc Curry, 2012). 
Según Justin Mc Curry (2012), conforme el día de la elección presidencial se acerca, la identidad del nuevo o la nueva dirigente del Sur continúa indefinida, pues según una encuesta reciente hecha por Real Meter la competencia entre Park y Moon es cerrada. Park cuenta con $47.8 \%$ de apoyo, mientras que Moon tiene $47.7 \%$.

Hasta el momento, la moneda sigue en el aire y aún no se sabe quién será el o la nueva dirigente del sur, por tanto, el tiempo será el que defina cuál será la tónica de las relaciones ínter-coreanas en por lo menos los próximos cinco años que dure el periodo presidencial en Corea del Sur.

\section{Anexos}

Anexo 1. Lista de presidentes de Corea del Sur y Corea del Norte

\begin{tabular}{|c|c|c|c|}
\hline \multicolumn{4}{|c|}{ Presidente/ Periodo de gobierno } \\
\hline \multicolumn{2}{|l|}{ República de Corea } & \multicolumn{2}{|c|}{$\begin{array}{l}\text { Republica Popular } \\
\text { Democrática de Corea }\end{array}$} \\
\hline \multicolumn{2}{|l|}{ Corea del Sur } & \multicolumn{2}{|c|}{ Corea del Norte } \\
\hline Rhee Syngman & 20 de julio 1948- 26 de abril 1960 & \multirow{8}{*}{$\begin{array}{l}\text { Kim Il- } \\
\text { sung }\end{array}$} & \multirow{8}{*}{$\begin{array}{l}28 \text { de diciembre } \\
\text { de } 1972-8 \text { de julio } \\
\text { de } 1994\end{array}$} \\
\hline Heo Jeong & 26 de abril 1960- 29 de julio 1960 & & \\
\hline Yun Po-sun & 29 de julio 1960- 16 de marzo 1961 & & \\
\hline Park Chung-hee & 15 de noviembre 1963- 26 de octubre 1979 & & \\
\hline Choi Kyu-ha & 26 de noviembre 1979- 27 de agosto 1980 & & \\
\hline Chun Doo-hwan & 2 de septiembre 1980- 25 de febrero 1988 & & \\
\hline Roh Tae-woo & 25 de febrero 1988- 25 de febrero 1993 & & \\
\hline Kim Young-sam & 25 de febrero 1993- 25 de febrero 1998 & & \\
\hline Kim Dae-jung & 25 de febrero 1998-25 de febrero 2003 & \multirow{4}{*}{$\begin{array}{l}\text { Kim } \\
\text { Jong-il }\end{array}$} & \multirow{4}{*}{$\begin{array}{l}8 \text { de julio de } \\
1994-17 \text { de } \\
\text { diciembre de } \\
2011\end{array}$} \\
\hline Roh Moo-hyun & 12 de marzo 2004- 14 de marzo 2004 & & \\
\hline Goh kun & 12 de marzo 2004- 14 de marzo 2004 & & \\
\hline Roh Moo-hyun & 14 de marzo 2004- 25 de febrero 2008 & & \\
\hline Lee Myung-bak & 25 de febrero 2008- 25 de febrero 2013 & $\begin{array}{l}\text { Kim } \\
\text { Jong-un }\end{array}$ & $\begin{array}{l}26 \text { de diciembre } \\
\text { de 2011-hasta } \\
\text { hoy }\end{array}$ \\
\hline
\end{tabular}


Anexo 2. Provocaciones norcoreanas hacia Corea del Sur (2000-2010)

\begin{tabular}{ll}
\hline Año & Acciones \\
\hline 2000 & A través de su señal de radio, Corea del Norte amenazó con volar en pedazos el diario \\
& conservador surcoreano Choson Ilbo por "calumniar a nuestra república" mediante el \\
reclamo de que la Guerra de Corea fue empezada por una invasión del sur al norte. \\
Corea del Norte arguyó que la acción del diario, perjudicial para la unidad nacional \\
y para la reunificación, "no es un asunto de libertad de prensa sino de alta traición". \\
Corea del Norte tildó al líder de oposición surcoreano, Lee Hoi-chang como "un \\
elemento anti-reunificación, un traidor, un idiota e imbécil". Lee fue acusado por \\
difamar la autoridad del liderazgo norcoreano cuando habló en la Asamblea Nacio- \\
nal surcoreana el seis de julio sobre la necesidad de reciprocidad en la cooperación \\
ínter-coreana.
\end{tabular}

2001 Botes patrulla norcoreanos entraban brevemente a aguas surocreanas del Mar Amarillo. Los barcos se retiraban después de ser retaos por barcos navales surcoreanos. Este tipo de intromisiones fue frecuente a lo largo del año.

En noviembre, soldados norcoreanos dispararon tres rondas a un puesto de guardia surcoreano, lo que llevo a los surcoreanos a responder los disparos. Nadie resultó herido.

2002 Intromisión marítima norcoreana a la isla de Yeongpyong en enero.

En junio, hubo intercambio de disparos entre barcos navales norcoreanos y surcoreanos. Presuntamente los barcos norcoreanos cruzaron los límites fronterizos y abrieron fuego a un buque patrulla surcoreano. Cuatro surcoreanos y un número indeterminado de norcoreanos murieron.

2003 A lo largo del año, Corea del Norte realizó pruebas de misiles al Mar del Este o Mar de Japón, además de irrumpir frecuentemente en aguas territoriales de Corea del Sur.

En febrero, Corea del Norte amenazó con abandonar el tratado del armisticio de 1953 si eu le imponía sanciones económicas, reclamando que el bloqueo económico y comercial violaría el artículo 15 del armisticio.

En febrero, un jet de combate norcoreano cruzó la zona aérea de Corea del Sur, lo que provocó que el sur enviara seis aviones de combate. Esta incursión del norte, que duró dos minutos, terminó sin incidentes, pero aumentó tensiones a tiempo que los nervios ya estaban puestos en el programa nuclear norcoreano. Esta incursión aérea no se había presentado desde de 1983.

En julio, hubo un intercambio de balas entre soldados norcoreanos y surcoreanos en la Zona Desmilitarizada.

2004- No hubo provocaciones directas de Corea del Norte hacia Corea del Sur, pero sí hubo 2007 pruebas de misiles y pruebas nucleares por parte de Corea del Norte, que para ese tiempo sus amenazas estaban dirigidas hacia Estados Unidos. 
2008 En marzo, el diálogo oficial entre ambas Coreas fue suspendido unilateralmente por Corea del Norte.

En abril, Corea del Norte lanzó agresiones verbales contra el presidente Lee Myungbak.

En julio, un soldado norcoreano disparó a muerte a una turista surcoreana cerca del complejo turístico del Monte Kumgang.

En noviembre, Corea del Norte rompió la línea telefónica de la aldea con tregua de Panmunjom.

En diciembre, Corea del Norte restringe los cruces transfronterizos.

2009 En enero, Corea del Norte comenzó con su postura de confrontación total.

En marzo, Corea del Norte interrumpió la línea de comunicación militar íntercoreana

En mayo, Corea del Norte condujo su segunda prueba nuclear

Finalmente, entre julio y agosto de este año, el régimen consideró letra muerta todos los acuerdos ínter-coreanos ya logrados, deslindándose de ellos.

2010 En enero, la comisión de defensa nacional norcoreana advirtió a Seúl que iniciaría una "guerra santa de represalias" contra las autoridades surcoreanas.

En noviembre. Bombardeo de la isla fronteriza surcoreana de Yeonpyong, donde murieron dos soldados y dos civiles surcoreanos.

\section{Anexo 3. Esquema del liderazgo norcoreano}
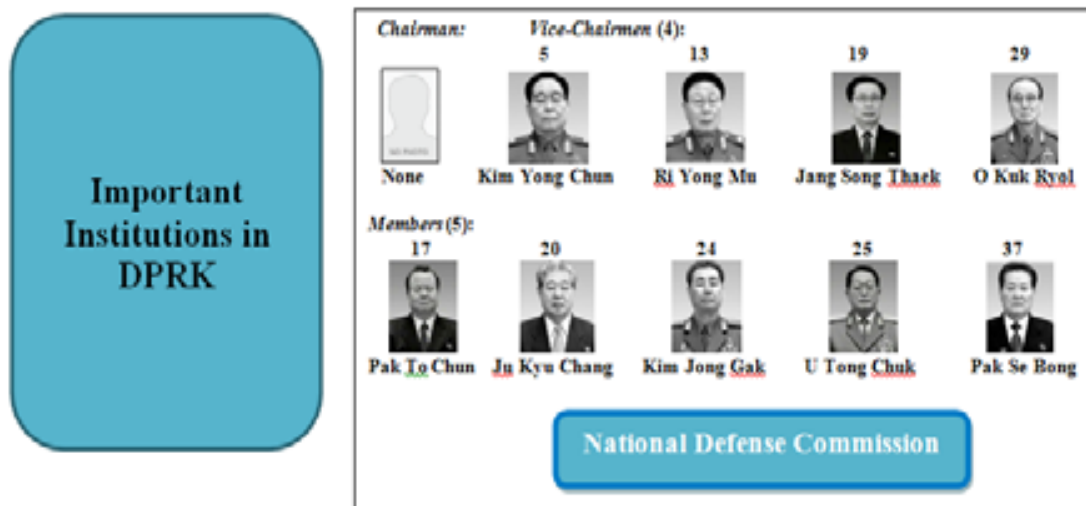

http://blog.keia.org/wp-content/uploads/2012/01/NK-Leadership-Part-11.png 

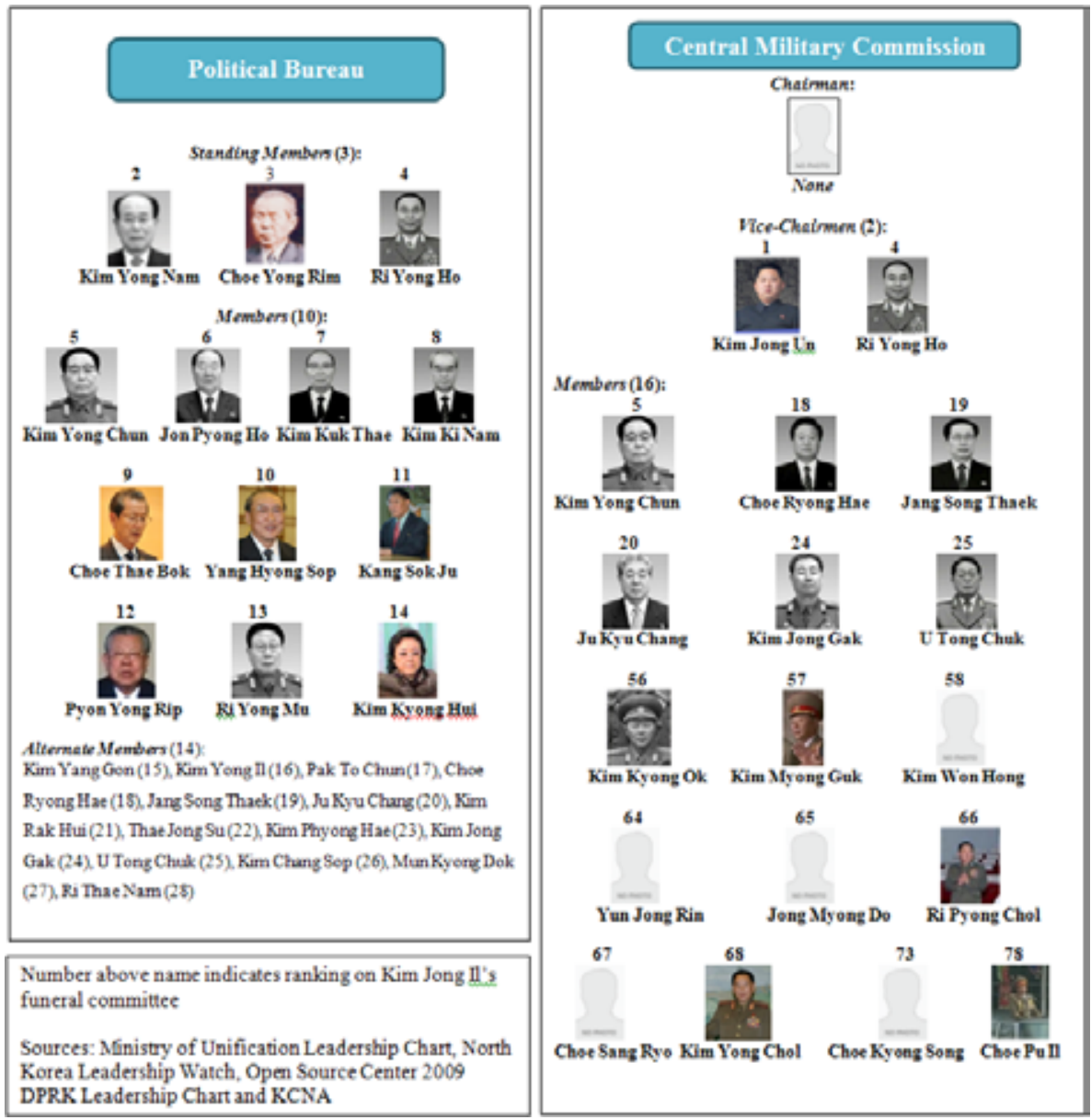

http://blog.keia.org/wp-content/uploads/2012/01/NK-Leadership-Part-21.png

\section{Referencias}

BBC (2011), “Profile: Kim Jong-un”, News Asia Pacific, (25 de febrero de 2012). Disponible en: http://www.bbc.co.uk/news/world-asia-pacific-11388628. Fecha de consulta: marzo 4, 2012.

Biblioteca del Congreso Nacional de Chile (BCN) (2012), 'Bajo la sombra de un conflicto', Dos voluntades para el desarrollo 50 años de relaciones entre Chile y Corea, Chile: Editorial Maval, p. 20, (septiembre de 2012). 
Disponible en: http://www.google.com/url?sa=t\&rct=j\&q=\&esrc=s\&sou rce=web\&cd=1\&cad=rja\&ved=0CDEQFjAA\&url=http\%3A\%2F\%2Fasiap acifico.bcn.cl\%2Fobtienearchivo\%3Fid\%3Ddocumentos\%2F10221.1\%2 F29264\%2F1\%2FLibroCoreaBCN.pdf\&ei=oNTNUIWYB4bKswbGvIHoB A\&usg=AFQjCNGdq7VRmgXLcODCU62Oodi4B1vguw\&bvm=bv.13553 25884,d.Yms. Fecha de consulta: diciembre 13, 2012.

CIA World Factbook (2012), North Korea Country Profile, Korea, North actualizado al 8 de febrero de 2012. Disponible en: https://www.cia.gov/ library/publications/the-world-factbook/geos/kn.html. Fecha de consulta: marzo 30, 2012.

Chung, Jae-ho (2001), "South Korea between Eagle and Dragon, Perceptual ambivalence and strategic dilemma", en Asian Survey (41:5). Berkley California: University of California Press, pp. 777-796.

CNN (2011), "EU y Japón esperan una transición pacífica del poder en Corea del Norte”. CNN México. Disponible en: http://mexico.cnn.com/mundo/2011/12/19/eu-y-japon-esperan-una-transicion-pacifica-del-poderen-corea-del-norte. Fecha de consulta: diciembre 19, 2011.

Del Arenal, Celestino (2007), 'Postulados del paradigma de la sociedad global', Introducción a las relaciones internacionales. España: Tecnos, Cuarta edición, pp. 33-34.

El Universal (2011), “Estados Unidos, Japón y Surcorea atentos tras muerte de Kim Jong-Il” El Universal Caracas. Disponible en: http://www.eluniversal. com/internacional/111219/estados-unidos-japon-y-surcorea-atentostras-muerte-de-kim-jong-il. Fecha de consulta: 19 de diciembre de 2011. El Confidencial (2011), "Pyongyang abre la era de Kim Jong-un en tono beligerante e inmovilista”, El Confidencial. Disponible en: http://www. elconfidencial.com/ultima-hora-en-vivo/2011/12/pyongyang-inaugurajongun-beligerante-inmovilista-20111230-654306.html. Fecha de consulta: diciembre 30, 2011.

Feffer, John (2011), “2012 sacudirá Asia y el mundo”, Revista Rebelión, (7 de octubre de 2011). Disponible en: http://www.rebelion.org/noticia. php?id=137048. Fecha de consulta: marzo 5, 2012.

Fischer, Hannah (2007), "North Korean Provocative Actions 1950-2007" Congressional Research Service, (20 de abril de 2007). Disponible en: http://assets.opencrs.com/rpts/RL30004_20070420.pdf. Fecha de consulta: agosto 15, 2012). 
Fuerza Aérea del Perú (s/f), El método sistémico. Disponible en: http://www. fap.mil.pe/instruccion/esfap/jetpo/docsPDF/MetodoSistemico.pdf. Fecha de consulta: marzo 3, 2012.

Hamisevicz, Nicholas, K. Yun, Sarah, O'Carroll, Chad; Stangarone, Troy (2012), "12 Things on the Korean Peninsula to Watch for in 2012", Korea Economic Institute, Inter-Korean, (11 de enero de 2012). Disponible en: http://blog.keia.org/2012/01/12-things-on-the-korean-peninsula-towatch-for-in-2012/. Fecha de consulta: abril 10, 2012.

Herman, Luke (2012), "North Korea Leadership Chart- Abridged", The Peninsula, a blog of Korea Economic Institute, North Korea, (5 de enero de 2012), Disponible en: http://blog.keia.org/2012/01/north-koreanleadership-chart-abridged/. Fecha de consulta: marzo 6, 2012.

Kim, Samuel y Kim, Abraham (2003), 'Conflict Management', en Mary Hawkesworth y Maurice Kogan (eds.) Encyclopedia of Government and Politics, New York: Routledge.

Kim, Samuel (2004), 'Introducction', en Samuel S. Kim (ed.) Inter-Korean relations: problems and prospects, New York: Palgrave Macmillan, pp. 1-19.

Kummetz, Pablo (2012), "Corea del Norte suspende enriquecimiento de uranio" Deutsche Weile Agencia de Noticias, (29 de febrero de 2012). Disponible en: http://www.dw.de/corea-del-norte-suspende-enriquecimientode-uranio/a-15775993. Fecha de consulta: marzo 3, 2012.

Lee, Sang-man (2001), "The directions and policy tasks of Inter-Korean Economic Cooperation after the Summits", International Conference commemorating centennial Korea-Belgium diplomatic relations, Center for European Integration Studies/ Center for Asia-Pacific Studies, Kyung Hee University, pp. 47-69.

Lyin Hyung-gu (2012) "Assessing political stability in post Kim Jong-il North Korea” Academic paper series, (4 de diciembre de 2012). Korea Economic Institute. Disponible en: http://www.keia.org/publication/assessingpolitical-stability-post-kim-jong-il-north-korea. Fecha de consulta: diciembre 13, 2012.

León Manriquez, José Luis (coord.) (2009) “Introducción”, Historia Mínima de Corea. México: El Colegio de México, pp. 11-22.

Lista de presidentes de Corea del Sur, Wikipedia http://es.wikipedia.org/wiki/ Anexo:Presidentes_de_Corea_del_Sur.Fecha de consulta: junio 30, 2012. Manyin, Mark y Nanto, Dick (2010), "The Kaesong North-South Korean Industrial Complex", BiblioGov. (1º de junio de 2010). Disponible en: http:// 
assets.opencrs.com/rpts/RL34093_20100601.pdf. Fecha de consulta: julio 30, 2012.

Manyin, Mark (2011), "Kim Jong-il's death: Implications for North Korea's stability and U.S policy", Congressional Research Service. Disponible en: http://www.fas.org/sgp/crs/row/R42126.pdf, 11 de enero de 2012. Fecha de consulta: agosto 15 de 2012.

Mc Curry, Justin (2012), "Park Geun-hye close to becoming South Korea's first female president", The Guardian, South Korea, (14 de diciembre de 2012). Disponible en: http://www.guardian.co.uk/world/2012/dec/14/ park-geun-hye-south-korea. Fecha de consulta: diciembre 15, 2012.

Ministry of Unification (2010) "The Lee Myung-bak Administration's North Korea Policy”, (17 de mayo de 2010). Disponible en: http:// eng.unikorea.go.kr/CmsWeb/viewPage.req?idx=PG0000000585\& boardDataId=BD0000218717\&CP0000000002_BO0000000101_ Action=boardView\&CP0000000002_BO0000000101_ViewName=board/ english/BoardView\&curNum=1. Fecha de consulta: julio 30, 2012.

Ojeda, Alfonso (2001), "Hacia un nuevo modelo de cooperación ínter-coreana", en Alfonso Ojeda, Ernesto de Laurentis y Álvaro Delgado (coords.) Corea frente a los desafíos del siglo XXI. Primer Simposio Internacional sobre Corea. Madrid, España: Centro Español de Investigaciones Coreanas (CEIC), pp. 51-67. Disponible en: http://www.florentinorodao.com/academico/ aca01a.pdf. Fecha de consulta: agosto 15, 2012.

RPP Internacional (2011), “Temen que Kim Jong-un intensifique la represión en Corea del Norte", RPP Internacional, Radio Programas del Perú por Internet, (20 de diciembre de 2011). Disponible en: http://www.rpp.com. pe/2011-12-20-temen-que-kim-jong-un-intensifique-la-represion-en-corea-del-norte-noticia_433247.html. Fecha de consulta: agosto 10, 2012. Yoon, Dae-kyu y Yang Moon-soo (2005), "Inter-korean economic cooperation for North Korean development: future challenges and prospects", Asia Perspectiva, vol. 29, núm. 3, 2005, pp. 5-30. Disponible en: http://www. asianperspective.org/articles/v29n3-a.pdf. Fecha de consulta: junio 30, 2012. 


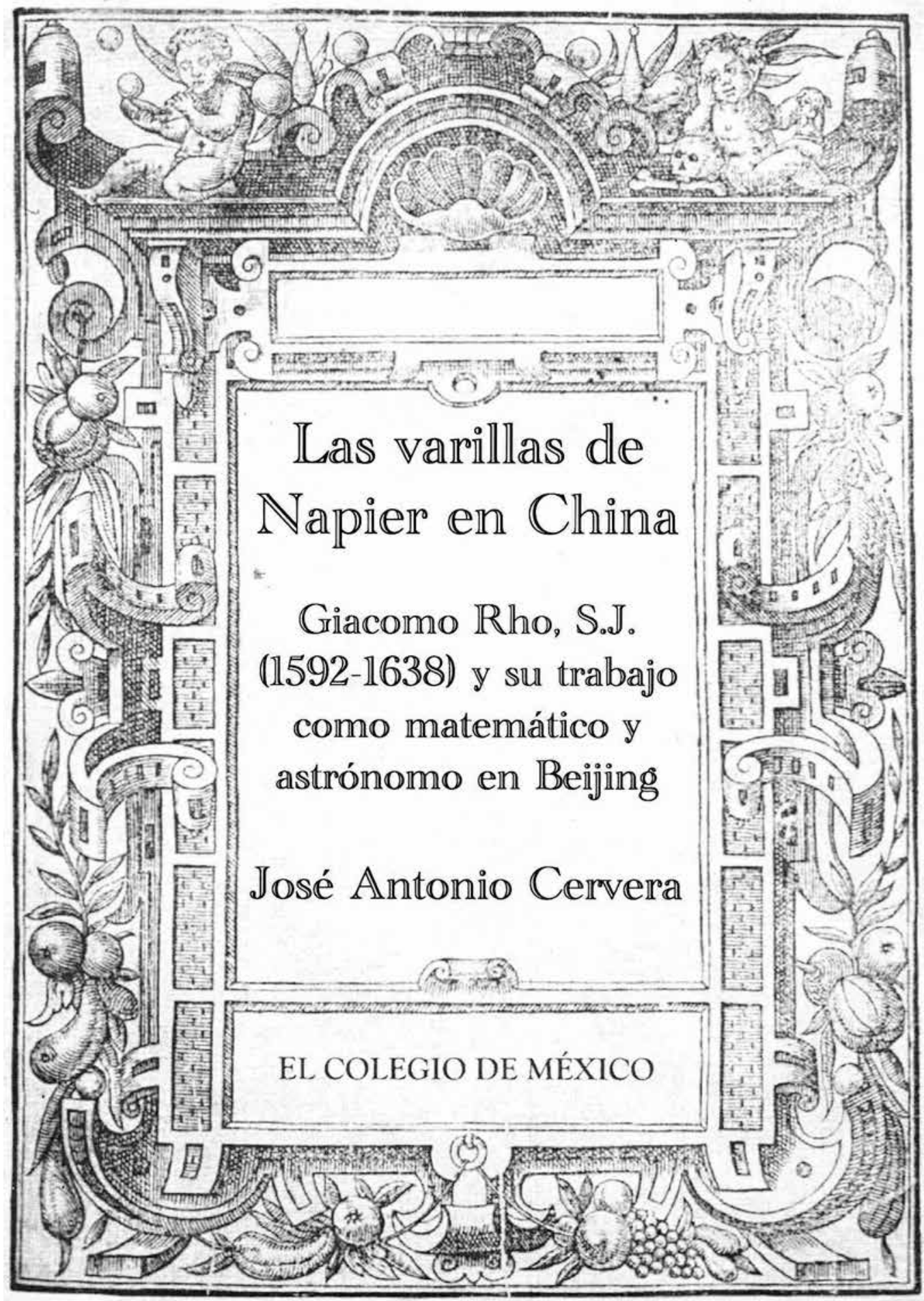

